\title{
Design and Analysis of CMOS Broad-Band Compact High-Linearity Modulators for Gigabit Microwave/Millimeter-Wave Applications
}

\author{
Hong-Yeh Chang, Member, IEEE, Pei-Si Wu, Student Member, IEEE, Tian-Wei Huang, Senior Member, IEEE, \\ Huei Wang, Fellow, IEEE, Chung-Long Chang, and John G. J. Chern
}

\begin{abstract}
CMOS broad-band compact high-linearity binary phase-shift keying (BPSK) and IQ modulators are proposed and analyzed in this paper. The modulators are constructed utilizing a modified reflection-type topology with the transmission lines implemented on the thick $\mathrm{SiO}_{2}$ layer to avoid the lossy silicon substrate. The monolithic microwave integrated circuit (MMIC) chips were fabricated using standard bulk 0.13- $\mu \mathrm{m}$ MS/RF CMOS process and demonstrated an ultracompact layout with more than $80 \%$ chip size reduction. The broadside couplers and $180^{\circ}$ hybrid for the modulators in the CMOS process are broad-band designs with low phase/amplitude errors. The dc offset and imbalance for the proposed topology are investigated and compared with the conventional reflection-type modulators. The measured dc offset was improved by more than $10 \mathrm{~dB}$. Both BPSK and IQ modulators feature a conversion loss of $13 \mathrm{~dB}$, a modulation bandwidth of wider than $1 \mathrm{GHz}$, and second- and third-order spur suppressions of better than $-30 \mathrm{dBc}$. The IQ modulator shows good sideband suppression with high local-oscillator suppression from 20 to $40 \mathrm{GHz}$. The modulators are also evaluated with a digital modulation signal and demonstrate excellent modulator quality and adjacent channel power ratio.
\end{abstract}

Index Terms-Binary phase-shift keying (BPSK), CMOS, coupler, millimeter wave (MMW), quadrature amplitude modulator (QAM).

\section{INTRODUCTION}

C MOS and SiGe direct-conversion modulators are commonly used in cellular phone and wireless local area network (WLAN) transmitters due to the low complexity and cost [1]-[6]. For high-level modulations, such as orthogonal frequency division multiplexing (OFDM) or n-quadrature amplitude modulation (n-QAM), high-linearity and low dc-offset binary phase-shift keying (BPSK) or IQ modulators are essential for the communication systems, and their performances have significant effects on the modulation quality [or signal-to-noise ratio (SNR)]. Generally, most of them employ ring-mixer or Gilbert-cell mixer configurations because of high local oscillator

Manuscript received May 23, 2005; revised September 6, 2005. This work was supported in part by the NTU-TSMC Joint-Development Project and the National Science Council of Taiwan, R.O.C., under Grant NSC 93-2752-E-002002-PAE, Grant NSC 93-2219-E-002-016, Grant NSC 93-2219-E-002-025, and Grant NSC 93-2213-E-002-033.

H.-Y. Chang, P.-S. Wu, T.-W. Huang, and H. Wang are with the Department of Electrical Engineering and the Graduate Institute of Communication Engineering, National Taiwan University, Taipei, 106 Taiwan, R.O.C. (e-mail: twhuang@cc.ee.ee.ntu.edu.tw).

C.-L. Chang and J. G. J. Chern are with the Taiwan Semiconductor Manufacturing Company, Hsinchu City, 300, Taiwan, R.O.C.

Digital Object Identifier 10.1109/TMTT.2005.860900
(LO)-to-RF isolation and good spurious suppression [7]. The CMOS Gilbert-cell-based modulators demonstrated excellent performance, but they are mostly below $10 \mathrm{GHz}$.

Conventional refection-type BPSK modulators, that consist of two bi-phase reflection-type modulators and two Lange couplers, were reported using InP- or GaAs-based high-electron mobility transistor (HEMT), and heterojunction bipolar transistor (HBT) monolithic microwave integrated circuit (MMIC) processes for the millimeter-wave (MMW) applications [8]-[12]. These modulators are usually broad-band with low LO drive and good amplitude/phase matches. However, the chip sizes of conventional reflection-type modulators are directly affected by the operation frequency. The conventional approach is relatively suitable for MMW MMIC designs. The other drawback of conventional reflection-type modulators is low LO-to-RF isolation (or high dc offset) due to the mismatch of components. From the previously reported results, the dc offset is severe in the constellation diagram and results a seriously LO leakage [10], but the LO leakage can be further improved with the additional dc-offset compensations [11].

To show the operation principle of the reflection-type BPSK and IQ modulators without imbalances and nonlinearity, the simplified time varying output voltage equations can be obtained from [11] and expressed as

$$
V_{\text {out }}^{\mathrm{BPSK}}(t)=K V_{B} \cos \left(\omega_{\mathrm{LO}} t\right)
$$

and

$$
V_{\text {out }}^{\mathrm{IQ}}(t)=K\left[V_{B I} \cos \left(\omega_{\mathrm{LO}} t\right)+V_{B Q} \sin \left(\omega_{\mathrm{LO}} t\right)\right]
$$

for BPSK and IQ modulators, respectively, where $K$ is modulation gain, $\omega_{\mathrm{LO}}$ is the angular frequency of the carrier signal, and $V_{B}, V_{B I}$, and $V_{B Q}$ are baseband signals. From (1), the BPSK modulation can be performed by sweeping $V_{B}$ from 1 to -1 (or -1 to 1 ) based on the baseband waveform. For the IQ modulator, the phase and amplitude of the carrier can be modulated by sweeping $V_{B I}$ and $V_{B Q}$ simultaneously, and the modulation schemes can be n-PSK or n-QAM based on the vector sum of the baseband inputs.

In [13], we proposed an innovative topology for the reflection-type BPSK modulator and implemented it using a $0.13-\mu \mathrm{m}$ CMOS process, which utilized a $180^{\circ}$ hybrid and a Wilkinson power combiner to replace the input and output $90^{\circ}$ hybrids. This modified reflection-type BPSK modulator features low dc offset, amplitude, and phase imbalances. In order to enhance the modulation bandwidth, the small inductors are used for RF 
choke at the gates of the NMOS devices and the small capacitors are used for RF bypass at the baseband input ports. In this paper, the proposed BPSK modulator is further applied to an IQ modulator design, and the quadrature LO signals are generated from a low-imbalance broadside coupler. The IQ modulator demonstrates a good modulation quality with wide modulation bandwidth. The broadside $90^{\circ}$ coupler and a very compact transformer with low amplitude and phase errors are also developed in the CMOS technology for the $90^{\circ}$ and $180^{\circ}$ hybrids used in the proposed reflection-type BPSK and IQ modulators. All passive elements are designed in thin-film microstrip line (or called microstrip-on-die in [14]). The layout of the thin-film microstrip line is very compact with low loss due to the thick $\mathrm{SiO}_{2}$ layer and the avoidance of the silicon substrate loss.

The imbalance analysis of the conventional reflection-type modulators presented the investigations of the phenomena versus the imbalances [11]. In this paper, we further analyze the dc offset and imbalance of the proposed reflection-type modulators. As compared with conventional reflection-type modulators, the proposed topology is less sensitive to the mismatch of the hybrids and device characteristics, as well as the amplitude and phase imbalances in the BPSK modulator. The couplers design of the proposed reflection-type modulator is more flexible, and the compact layouts are easily achieved with the tradeoff between the imbalances in the two $90^{\circ}$ couplers using CMOS technology. According to the experimental results, the BPSK and IQ modulators feature low conversion loss and high linearity with ultra compact chip sizes. To the best of our knowledge, this is the first attempt to use CMOS technology to demonstrate BPSK and IQ modulators by using the modified reflection-type topology in the MMW regime with much smaller sizes $\left(0.5 \times 0.35 \mathrm{~mm}^{2}\right.$, $0.65 \times 0.58 \mathrm{~mm}^{2}$ ) and gigabit modulation bandwidth.

\section{OPERATION PRINCIPLE AND ANALYSIS}

\section{A. BPSK Modulator}

From our prior work, the conventional reflection-type BPSK modulator using GaAs HBT technology was reported in [11], and it is configured in a balanced structure similar to that of the balanced amplifiers. Based on the reflection-type topology, the block diagram of the proposed modified reflection-type BPSK modulator is shown in Fig. 1(a), which comprises a transformer, an in-phase combiner, and two bi-phase reflection modulators. $\mathrm{BB}+$ and $\mathrm{BB}-$ are differential input ports for the baseband signal. The bi-phase reflection modulator consists of a $90^{\circ}$ hybrid and two NMOS devices. Assuming that the $90^{\circ}$ hybrid has a phase error of $\theta_{e}$ and an amplitude error of $A_{e}$, the transformer has a phase error of $\theta_{e t}$ and an amplitude error of $A_{e t}$, and the in-phase combiner has a phase error of $\theta_{e w}$ and an amplitude error of $A_{e w}\left(A_{e}, A_{e t}\right.$, and $A_{e w} \geq 1$, while $A_{e}, A_{e t}$, and $A_{e w}=1$ for the ideal case). The transmission coefficients $S_{21}$ of the modified reflection-type BPSK modulator can be expressed as

$$
S_{21}^{\mathrm{BPSK}}=\frac{1}{2} \frac{e^{j\left(\frac{\pi}{2}+\theta_{e}\right)}}{A_{e}}\left[\frac{\Gamma_{m}}{A_{e w} A_{e t}} e^{j\left(\theta_{e w}+\theta_{e t}\right)}+\bar{\Gamma}_{m} e^{j \pi}\right]
$$

where $\Gamma_{m}$ is the input reflection coefficient looking into the drain of the NMOS at the BB+ port (device is turned on or off), and $\bar{\Gamma}_{m}$ is at the BB- port (device is turned off or on). There are two phase states for the BPSK operation: the devices

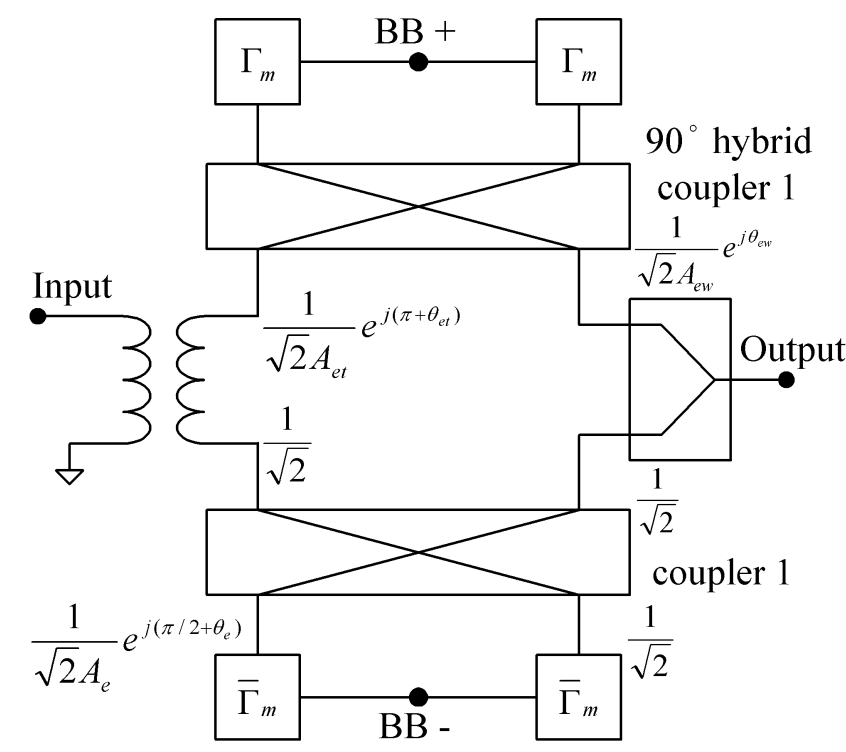

(a)

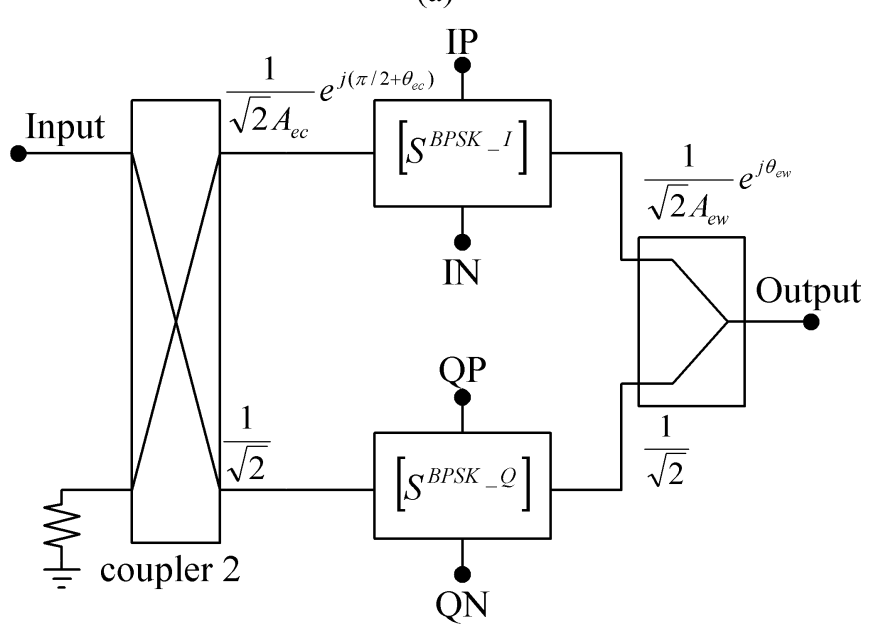

(b)

Fig. 1. Block diagram of the modified reflection-type modulators. (a) BPSK modulator. (b) IQ modulator.

at $\mathrm{BB}+$ port is off and the devices at $\mathrm{BB}-$ port is on for the state- 0 , while the devices at $\mathrm{BB}+$ port is on and the devices at $\mathrm{BB}-$ port is off for the state-1. Assuming that the amplitude error $A_{e}$ and $A_{e t}$ are $\sqrt{2}(3 \mathrm{~dB})$, and the phase error $\theta_{e}$ and $\theta_{e t}$ are $5^{\circ}(\pi / 36 \mathrm{rad})$. Since the in-phase combiner is a symmetric architecture, we suppose that the amplitude imbalance $A_{e w}$ is 1 and the phase error $\theta_{e w}$ is 0 . The state- 0 transmission coefficient $S_{21}^{0}$ of the BPSK modulator can be expressed as

$$
S_{21}^{0}=\frac{1}{2 \sqrt{2}} e^{j\left(\frac{19 \pi}{36}\right)}\left[\frac{\left(1-M_{d}\right) e^{j\left(\theta_{d}\right)}}{\sqrt{2}} e^{j\left(\frac{\pi}{18}\right)}+e^{j \pi}\right]
$$

and the state-1 transmission coefficient $S_{21}^{1}$ can be expressed as

$$
S_{21}^{1}=\frac{1}{2 \sqrt{2}} e^{j\left(\frac{19 \pi}{36}\right)}\left[\frac{1}{\sqrt{2}} e^{j\left(\frac{\pi}{18}\right)}+\left(1-M_{d}\right) e^{j\left(\theta_{d}+\pi\right)}\right]
$$

where $M_{d}$ is the magnitude difference between $\Gamma_{m}$ and $\bar{\Gamma}_{m}$ and $\theta_{d}$ is the phase difference between $\Gamma_{m}$ and $\bar{\Gamma}_{m}\left(M_{d}=0\right.$, $\theta_{d}=180^{\circ}$ for ideal case). For the conventional reflection-type BPSK modulator, the transmission coefficients $S_{21}$ with imbalances can be found in [11]. Similarly, we assume that the Lange coupler has an amplitude error of $\sqrt{2}(3 \mathrm{~dB})$ and a phase error 


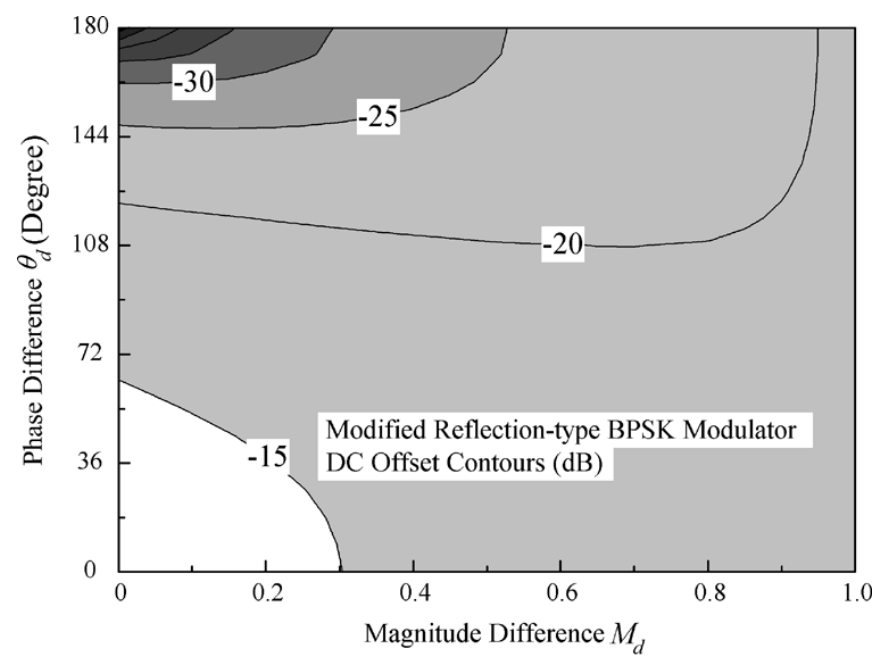

(a)

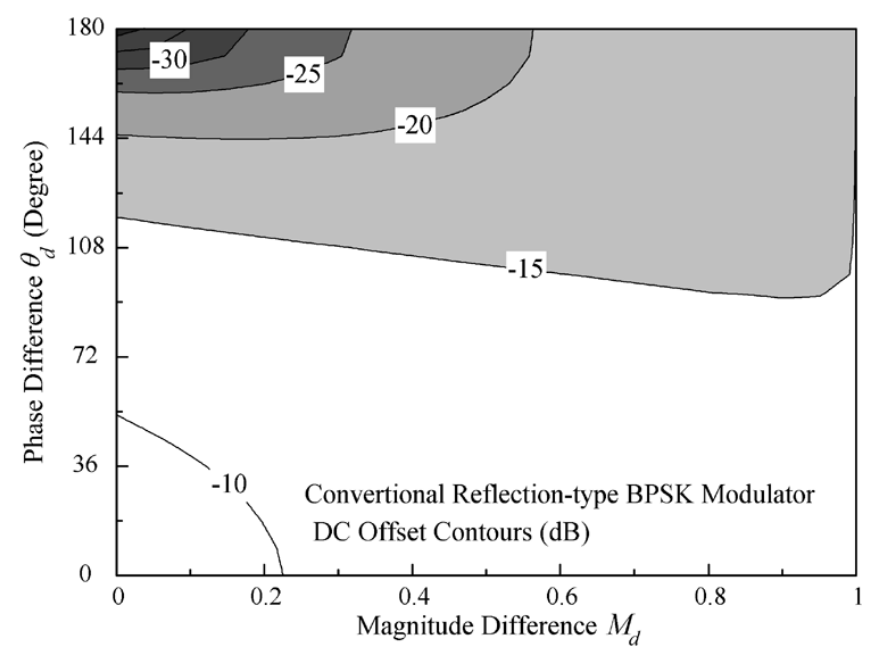

(b)

Fig. 2. DC offset contours versus the magnitude difference $M_{d}$ and the phase difference $\theta_{d}$. (a) Modified reflection-type BPSK modulator. (b) Conventional reflection-type BPSK modulator.

of $5^{\circ}$. The state- 0 transmission coefficient $S_{C 21}^{0}$ of the conventional reflection-type BPSK modulator can be expressed as

$$
S_{C 21}^{0}=\frac{1}{2 \sqrt{2}}\left[\frac{\left(1-M_{d}\right) e^{j\left(\theta_{d}\right)}}{2} e^{j\left(\frac{3 \pi}{2}+\frac{3 \pi}{36}\right)}+e^{j\left(\frac{\pi}{2}+\frac{\pi}{36}\right)}\right]
$$

and the state- 1 transmission coefficient $S_{C 21}^{1}$ can be expressed as

$$
S_{C 21}^{1}=\frac{1}{2 \sqrt{2}}\left[\frac{1}{2} e^{j\left(\frac{3 \pi}{2}+\frac{3 \pi}{36}\right)}+\left(1-M_{d}\right) e^{j\left(\theta_{d}+\frac{\pi}{2}+\frac{\pi}{36}\right)}\right] .
$$

The dc offset of the BPSK modulator can be expressed as

$$
\text { DC_offset }=20 \log \left[\operatorname{Mag}\left(S_{21}^{0}+S_{21}^{1}\right)\right]
$$

and the dc offset is zero for ideal case. The dc offset contours in decibel versus the magnitude difference $M_{d}$ and the phase difference $\theta_{d}$ are plotted in Fig. 2 for the modified and the conventional reflection-type BPSK modulators. The magnitude difference $M_{d}$ is swept from 0 to 1 , and the phase difference $\theta_{d}$ is swept from $0^{\circ}$ to $180^{\circ}$. As can be observed, the dc offset of the proposed topology is less sensitive to the mismatch of the device characteristics. In most of the area of Fig. 2(a), the dc offset is

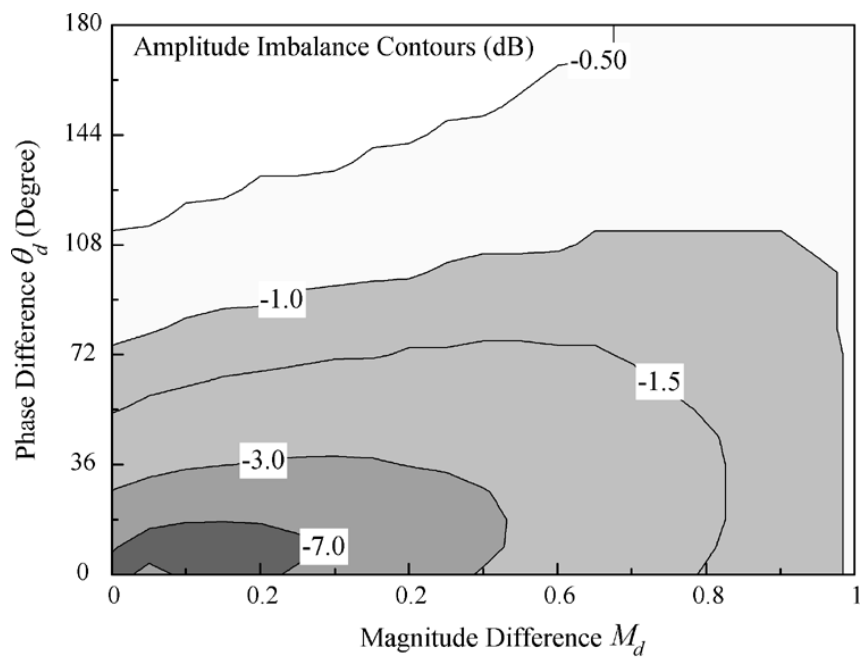

(a)

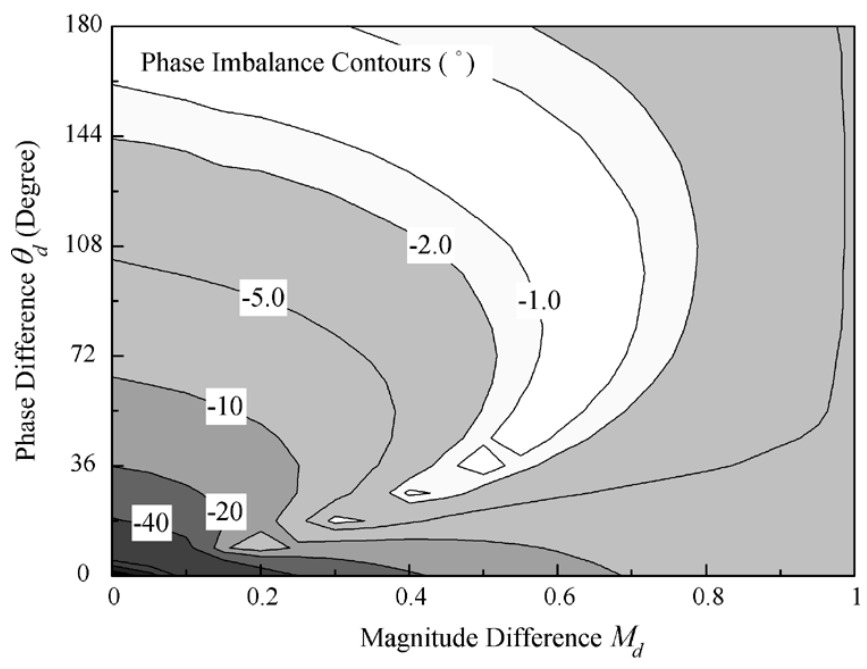

(b)

Fig. 3. Imbalances versus the magnitude difference $M_{d}$ and the phase difference $\theta_{d}$ for the modified reflection-type BPSK modulator. (a) Amplitude imbalance contours. (b) Phase imbalance contours between states 0 and 1 $\left(A_{e}=3 \mathrm{~dB}, A_{e t}=1 \mathrm{~dB}, \theta_{e}=5^{\circ}\right.$, and $\left.\theta_{e t}=5^{\circ}\right)$.

better than $-15 \mathrm{~dB}$. From the EM simulation and the experimental results of the $180^{\circ}$ hybrid, the amplitude error $A_{e t}$ is within $1.122(1 \mathrm{~dB})$, therefore, the dc offset should be better than $-20 \mathrm{~dB}$. On the contrary, the dc offset of the conventional reflection-type BPSK modulator is only about $-10 \mathrm{~dB}$, as shown in Fig. 2(b). It can be observed that the proposed reflection-type BPSK modulator is easier to match to the device and can be further used for broad-band circuit designs with low dc offset.

For the imbalance analysis of the modified reflection-type BPSK modulator, the imbalance contours between states 0 and 1 are plotted in Fig. 3 for amplitude and phase imbalances. In most of the area of the charts, the amplitude imbalance is within $1.5 \mathrm{~dB}$ and the phase imbalance is within $5^{\circ}$. From (3) and the imbalance investigation, the insertion losses of the modified BPSK modulator degrade with the amplitude imbalance $A_{e}$ increasing, but the phase error $\theta_{e}$ causes only phase delay without any degradation. In the case where the magnitude difference $M_{d}$ is within 0.5 and the phase difference $\theta_{d}$ is greater than $170^{\circ}$, the amplitude and phase imbalances of the modified reflection-type 
TABLE I

COMPARISON OF MAXIMUM AMPLITUDE AND PHASE IMBALANCES FOR THE MODIFIED AND CONVENTIONAL BPSK MODULATORS

$$
\left(M_{D} \leqq 0.5, \theta_{D} \geqq 170^{\circ}\right)
$$

\begin{tabular}{l|c|c}
\hline \hline & $\begin{array}{c}\text { Maximum Amplitude } \\
\text { Imbalance }(\mathrm{dB})\end{array}$ & $\begin{array}{c}\text { Maximum Phase } \\
\text { Imbalance }\left(^{\circ}\right)\end{array}$ \\
\hline Modified Modulator & 5.5 \\
$A_{e t}: 1 \sim 2, \theta_{e t}: 0^{\circ} \sim 20^{\circ}$ & 2 & \\
$A_{e}: 1 \sim 8, \theta_{e}: 0^{\circ} \sim 50^{\circ}$ & & 10.2 \\
\hline Conventional Modulator & 2.5 & \\
$A_{e}: 1 \sim 2, \theta_{e}: 0^{\circ} \sim 20^{\circ}$ & & \\
\hline \hline
\end{tabular}

BPSK modulator are almost within $2 \mathrm{~dB}$ and $5^{\circ}$, when the amplitude errors $A_{e}$ and $A_{e t}$ are $\sqrt{2}(3 \mathrm{~dB})$ and the phase errors $\theta_{e}$ and $\theta_{\text {et }}$ are $20^{\circ}$.

Comparing with the conventional reflection-type BPSK modulator, the maximum amplitude and phase imbalances are listed in Table I. The modified reflection-type BPSK modulator obviously features lower amplitude/phase imbalances, even when the amplitude error $A_{e}$ is up $8(18 \mathrm{~dB})$ and the phase error $\theta_{e}$ is up to $50^{\circ}$. Therefore, the modified reflection-type modulator also has better robustness to the amplitude/phase errors of the hybrids. When the amplitude errors $A_{e t}$ is greater than $\sqrt{2}(3 \mathrm{~dB})$, the phase error $\theta_{e t}$ is greater than $20^{\circ}$, and the phase difference $\theta_{d}$ is smaller than $170^{\circ}$, the errors $\left(A_{e t}\right.$ and $\left.\theta_{e t}\right)$ of the $180^{\circ}$ hybrid will be strongly related to the amplitude/phase imbalances of the modulator, and the imbalances also can be obtained from the imbalance calculation in (3). From above discussions, we can summarize that i) the insertion losses of the modulator are affected by the magnitude/phase of the input reflection coefficient, the amplitude imbalance of the $90^{\circ}$ hybrid (coupler 1), and the losses of the passive components; ii) the imbalances of the modulator will be dominated by the performance of the $180^{\circ}$ hybrid.

\section{B. IQ Modulator}

The block diagram of the IQ modulator is shown in Fig. 1(b), which employs two modified reflection-type BPSK modulators, a $90^{\circ}$ hybrid (coupler 2), and an in-phase combiner. IP/IN and QP/QN are differential baseband signal input ports for BPSK I and Q channels, respectively. Since the imbalances of coupler 2 seriously affect the quadrature error and amplitude imbalance of the IQ modulator, we assume that coupler 2 has an amplitude imbalance of $A_{e c}$ and a phase error of $\theta_{e c}$; these notations are different from the imbalances of coupler 1 discussed in the previous section on the BPSK modulator. The transmission coefficients $S_{21}$ of the modified reflection-type IQ modulator can be expressed as

$$
\begin{aligned}
S_{21}^{\mathrm{IQ}}= & \frac{1}{4} \frac{e^{j\left(\frac{\pi}{2}+\theta_{e}\right)}}{A_{e}} \\
& \times\left\{\frac{e^{j\left(\theta_{e c}+\theta_{e w}\right)}}{A_{e c} A_{e w}}\left[\frac{\Gamma_{I}}{A_{e w} A_{e t}} e^{j\left(\frac{\pi}{2}+\theta_{e w}+\theta_{e t}\right)}+\bar{\Gamma}_{I} e^{j\left(\frac{3 \pi}{2}\right)}\right]\right. \\
& \left.\quad\left[\frac{\Gamma_{Q}}{A_{e w} A_{e t}} e^{j\left(\theta_{e w}+\theta_{e t}\right)}+\bar{\Gamma}_{Q} e^{j(\pi)}\right]\right\}
\end{aligned}
$$

where $\Gamma_{I} / \bar{\Gamma}_{I}$ and $\Gamma_{Q} / \bar{\Gamma}_{Q}$ are the input reflection coefficients looking into the drain of the NMOS at the I- and Q-channel BPSK modulators, respectively. From (9), we can see that the insertion loss is affected by the amplitude imbalance $A_{e}$ of coupler 1 , and some phase delay without any distortion is caused by the phase error $\theta_{e}$. These phenomena are similar to the results discussed for the BPSK modulator. For the imbalances of coupler 2 and the in-phase combiner, the quadrature error degrades with increasing the phase imbalances of $\theta_{e c}$ and $\theta_{e w}$, and the I/Q channel imbalance increases with increasing the amplitude imbalances of $A_{e c}$ and $A_{e w}$. Generally, the amplitude/phase errors of the in-phase combiner are very low due to its symmetric structure, and therefore they can be neglected for these investigations. For the case of low mismatch among the devices, coupler 2, and the in-phase combiner, the insertion loss of the IQ modulator increases as the amplitude imbalance $A_{e t}$ of the $180^{\circ}$ hybrid deteriorates, while the phase error $\theta_{\text {et }}$ also causes only phase delay without any distortion. Otherwise, the imbalances of the $180^{\circ}$ hybrid affect quadrature error, I/Q channel amplitude, and dc offset of the IQ modulator. From the above-mentioned discussion, the $180^{\circ}$ hybrid and the $90^{\circ}$ coupler are strongly related to the performances of the IQ modulator. In order to reduce the distortion and imbalances of the IQ modulator, the imbalances of the $180^{\circ}$ hybrid and coupler 2 should be minimized.

\section{CirCuit DESIGN}

The broad-band modulators were designed using a TSMC commercial standard bulk $0.13-\mu \mathrm{m}$ 1P8M CMOS process, which provides one poly layer for the gates of CMOS transistors and eight metal layers for interconnections. The metal-insulator-metal (MIM) capacitor, spiral inductor, and polysilicon resistors with several $\Omega / \square$ and $\mathrm{k} \Omega / \square$ are available in this process. The active device (NMOS) exhibits a unit current gain frequency $\left(f_{T}\right)$ of $90 \mathrm{GHz}$ and a maximum oscillation frequency $\left(f_{\max }\right)$ of $106 \mathrm{GHz}$ with a $1.2-\mathrm{V}$ supply. In order to avoid the silicon substrate loss, the metal 1 (bottom layer) in the 1P8M CMOS process is used for ground plane, and the metal 8 (top layer) is used for the microstrip line with the thick $\mathrm{SiO}_{2}$ layer as substrate. The internal metal layers (metals 2-7) are used for broadside-coupler design and interconnections.

\section{A. Compact $90^{\circ}$ and $180^{\circ}$ Couplers in the CMOS Process}

The $90^{\circ}$ hybrids used for bi-phase reflection modulator are implemented using broadside-coupled lines, because the gap of the edge coupler is too small to fabricate for the required coupling. They are meandered to achieve a compact layout, which is another advantage of the broadside coupler compared with the Lange coupler. The layout and cross section of coupler 1 is shown in Fig. 4(a) with an area of $196 \times 40 \mu \mathrm{m}^{2}$. The space and width of the striplines first can be obtained from the required even- and odd-mode characteristic impedances, and then the coupling coefficients are calculated using the full-wave EM simulator SONNET [15]. Metal layers 7and 8 are used for the coupled lines of the broadside coupler due to the thick dielectric layer, and metal 1 is also used for the ground plane to keep away from the Si substrate. The line width $(w)$ is $4 \mu \mathrm{m}$ with a line length of approximately $300 \mu \mathrm{m}$. The thickness of the $\mathrm{SiO}_{2}$ layer between metals 1 and 7 is represented by $h_{1}$ with a dielectric constant of $\varepsilon_{r 1}$ and between metals 7 and 8 is represented by $h_{2}$ with a dielectric constant of $\varepsilon_{r 2}$. From the analysis in Section II, the imbalances of coupler 1 has little effect on the 


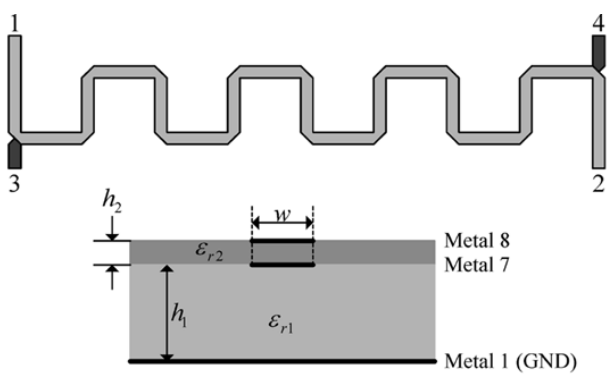

(a)

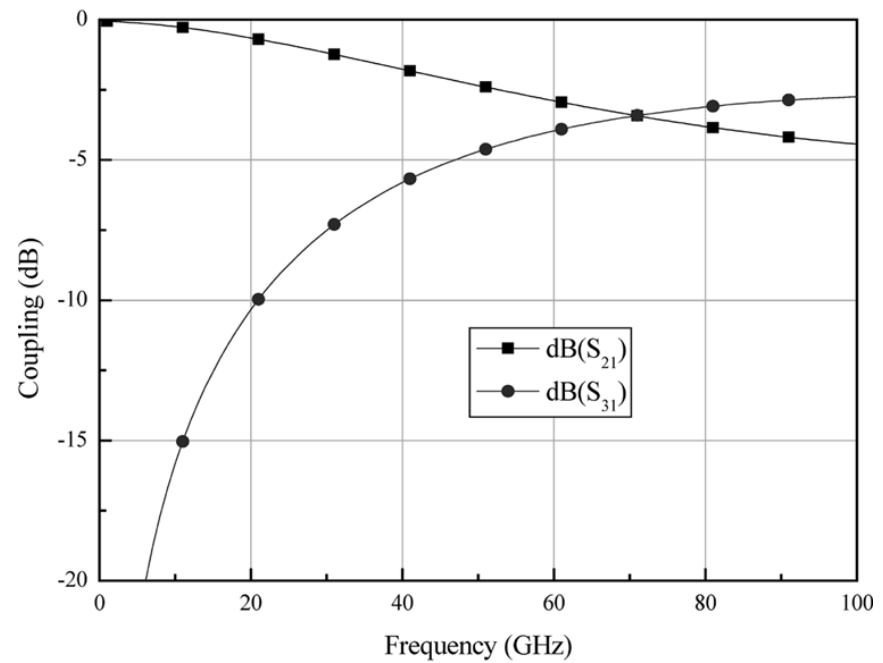

(b)

Fig. 4. Coupler 1. (a) Layout and cross section with an area of $196 \times 40 \mu \mathrm{m}^{2}$. (b) Simulated results.

performance of the modified reflection-type BPSK modulator (except for the insertion loss), and thus the center frequency of the coupler can be designed higher to make the coupler compact. The simulated results of coupler 1 are plotted in Fig. 4(b) from 1 to $100 \mathrm{GHz}$. The coupled port has a maximum coupling of $2.7 \mathrm{~dB}$ at a center frequency $(100 \mathrm{GHz})$. The average coupling for the direct and coupled ports is $3.5 \mathrm{~dB}$ between $50-100 \mathrm{GHz}$. The phase difference between the direct and coupled ports is approximately $90^{\circ}$ with an error within $3^{\circ}$ in the entire band.

The layout and cross-sectional view of coupler 2 is illustrated in Fig. 5(a) with an area of $340 \times 65 \mu \mathrm{m}^{2}$. The broadside coupled lines are composed of metals 7 and 8 , and the line width is $4 \mu \mathrm{m}$ with a line length of approximately $850 \mu \mathrm{m}$. From Section II, the performance of the IQ modulator is seriously degraded by the imbalances of coupler 2 , and coupler 2 should be carefully designed. To obtain the appropriate coupling, we add an additional offset distance ( $d$ of $1 \mu \mathrm{m}$ ) between metals 7 and 8 to reduce the coupling coefficient. The coupling can be reduced by decreasing the line width, but the insertion loss will increase due to the metal loss and mismatch. The simulated results of coupler 2 are plotted in Fig. 5(b), which features a maximum coupling of $2.7 \mathrm{~dB}$ at a center frequency of $45 \mathrm{GHz}$, an amplitude imbalance of within $2 \mathrm{~dB}$, and a phase imbalance of within $2^{\circ}$ between $20-70 \mathrm{GHz}$. The average coupling coefficient for the direct and coupled ports is about $3.7 \mathrm{~dB}$.

In the design of the $180^{\circ}$ hybrid, a Marchand balun [7] is used as the $180^{\circ}$ hybrid due to its excellent amplitude/phase match and broad-band response. The layout and cross section of the $180^{\circ}$ hybrid is shown in Fig. 6. Two coupled lines in the Marc-

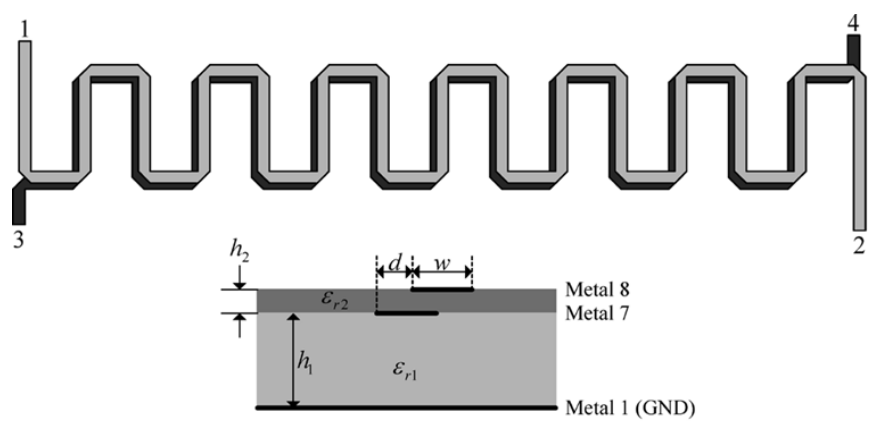

(a)

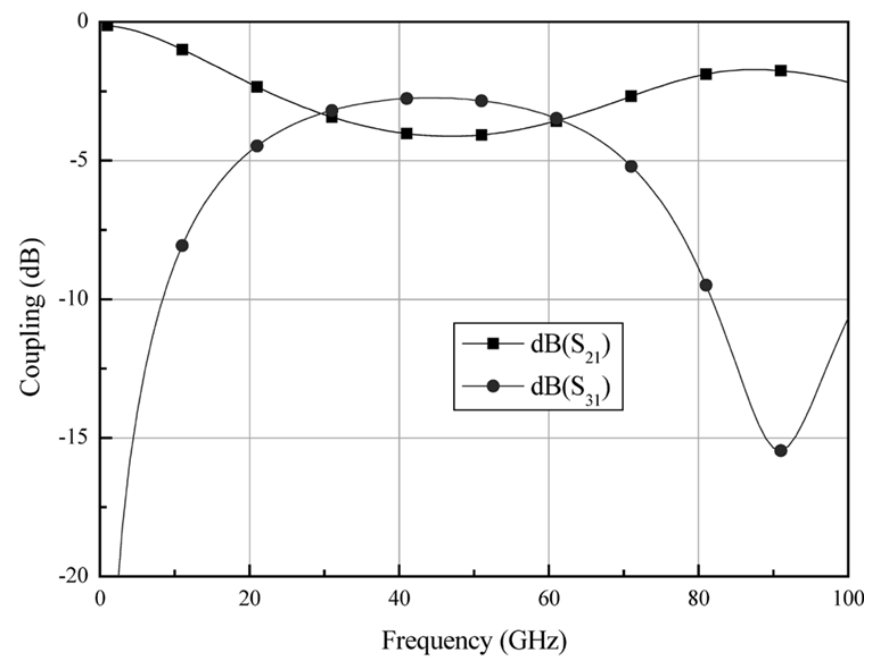

(b)

Fig. 5. Coupler 2. (a) Layout and cross section with an area of $340 \times 65 \mu \mathrm{m}^{2}$. (b) Simulated results.
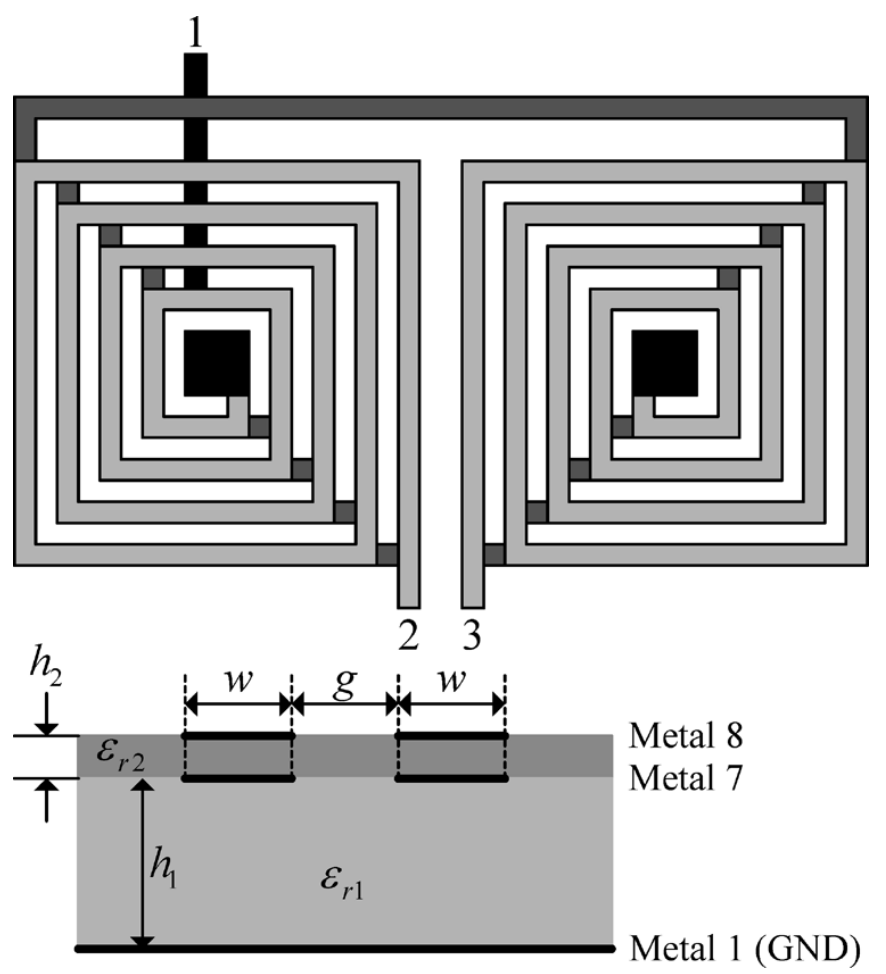

Fig. 6. Layout and cross section of the $180^{\circ}$ hybrid with an area of $120 \times$ $65 \mu \mathrm{m}^{2}$.

hand balun are also constructed of broadside-coupled lines with the space and width of the transmission lines obtained from the 


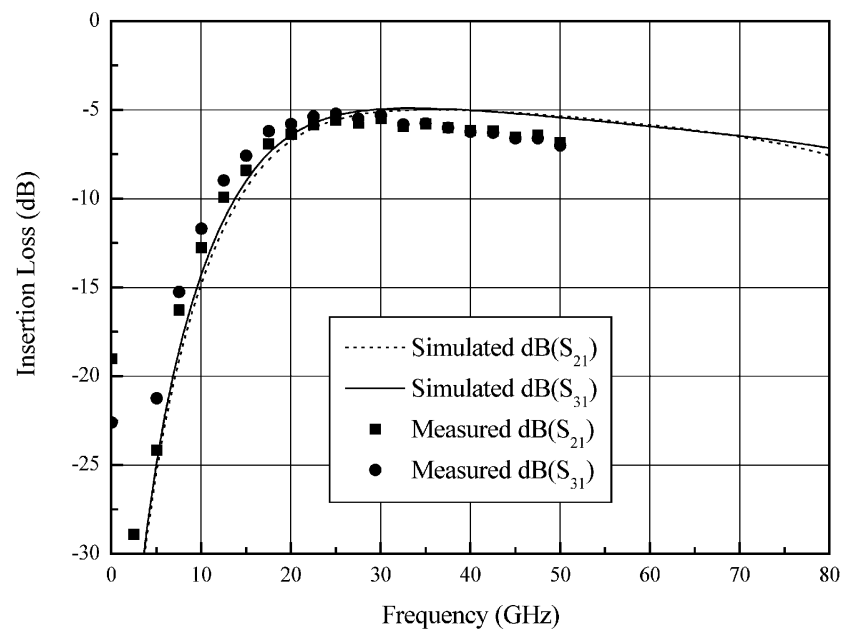

(a)

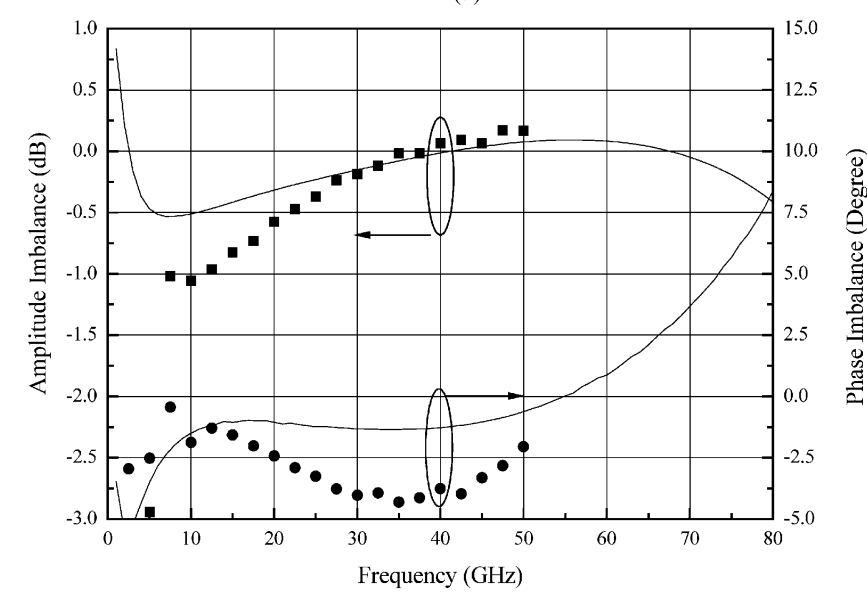

(b)

Fig. 7. Measured and simulated results of the $180^{\circ}$ hybrid. (a) Insertion losses. (b) Amplitude and phase imbalance (simulation: solid line; measurement: symbols).

required even- and odd-mode characteristic impedances. These two coupled lines are wound into a very compact area of $120 \times$ $65 \mu \mathrm{m}^{2}$, and the structure is similar to the spiral broadside coupled striplines (SBCSs) using low-temperature co-fired ceramic (LTCC) [16] and GaAs processes [17]. The line width $(w)$ and gap $(g)$ are both $3 \mu \mathrm{m}$. The measured (symbol) and simulated (solid line) insertion losses of the $180^{\circ}$ hybrid are plotted in Fig. 7(a), which features a simulated insertion loss of better than $8 \mathrm{~dB}$ between $15-80 \mathrm{GHz}$. The measured results are obtained from the port reduction method [18] that extracts the three-port $S$-parameters from multiple sets of two-port measurement results. The measured and simulated amplitude/phase imbalances versus frequency are plotted in Fig. 7(b). It is observed that the measured amplitude imbalance is within $1 \mathrm{~dB}$, the measured phase imbalance is within $5^{\circ}$ from 15 to $50 \mathrm{GHz}$, and the measured results agree with the simulated results.

\section{B. BPSK and IQ Modulators}

The schematic of the modified reflection-type BPSK modulator is shown in Fig. 8, and the photograph of the modified reflection-type BPSK modulator was shown in [13] with a chip size of $0.5 \times 0.35 \mathrm{~mm}^{2}$. For optimal design of the modulator, the device size of the NMOS is properly selected based on the

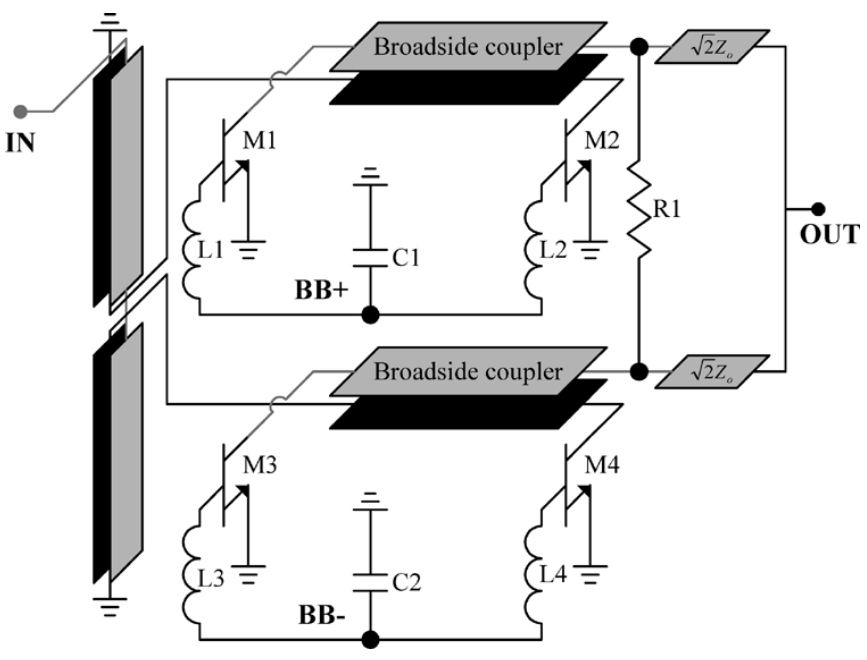

Fig. 8. Schematic of the modified reflection-type BPSK modulator.

on- and off-state reflection coefficients. A Wilkinson combiner is used for the in-phase combiner, and the quarter-wave transmission lines in the combiner are also meandered to achieve a compact layout. In order to enhance the modulation bandwidth, small inductors and capacitors are used for a low-pass network at the baseband input ports. The biases are $1 \mathrm{~V}$ at $\mathrm{BB}+$ and 0 $\mathrm{V}$ at $\mathrm{BB}-$ for state 0 ; the biases are $0 \mathrm{~V}$ at $\mathrm{BB}+$ and $1 \mathrm{~V}$ at $\mathrm{BB}-$ for state 1 . For the state OFF of the modulator, the biases at $\mathrm{BB}+$ and $\mathrm{BB}-$ are both $0.5 \mathrm{~V}$. The simulated results of the modified reflection-type BPSK modulator will be shown in Section IV and compared with the measured results. Between 18-80 $\mathrm{GHz}$, the simulated insertion losses for states 0 and 1 are better than $12 \mathrm{~dB}$ with an amplitude imbalance of within $1 \mathrm{~dB}$ and a phase imbalance of within $5^{\circ}$, and the simulated insertion loss for state OFF is better than $35 \mathrm{~dB}$. The conversion loss versus the LO frequency for the modulator is also simulated from 10 to $50 \mathrm{GHz}$ when it performs as a double-sideband up-converter mixer. The simulated conversion loss is better than $10 \mathrm{~dB}$ with an output $P_{1 \mathrm{~dB}}$ of higher than $-5 \mathrm{dBm}$ from 20 to $50 \mathrm{GHz}$. The baseband signal is a $5-\mathrm{MHz}$ single-tone signal with a power of $-8 \mathrm{dBm}$, and the LO drive power is $5 \mathrm{dBm}$.

The schematic of the modified reflection-type IQ modulator is shown in Fig. 9, which utilizes a $90^{\circ}$ coupler (coupler 2) to generate the quadrature-phase signals for the I and $Q$ paths and uses an in-phase combiner to combine the I/Q modulated signals. The photograph of the modulator is shown in Fig. 10 with a chip size of $0.65 \times 0.58 \mathrm{~mm}^{2}$. The metal 2 is used for the interconnection between baseband input pads and the nodes of IP, IN, QP, and QN. The baseband interconnections are properly routed to keep away from the RF or LO signal. The nodes of IP, IN, QP, and QN are usually biased with a dc voltage of $0.5 \mathrm{~V}$ to enhance the LO-to-RF isolation, when the circuit is performed as a mixer or modulator. The conversion loss versus LO frequency for the IQ modulator is simulated and presented in Section IV from 15 to $40 \mathrm{GHz}$ when it is performed as an image-rejection up-converter mixer. The IF (baseband) input is a 5-MHz single-tone signal with a power of $-10 \mathrm{dBm}$, and the $\mathrm{LO}$ drive power is $10 \mathrm{dBm}$. Between 20 to $40 \mathrm{GHz}$, the simulated conversion loss is better $11 \mathrm{~dB}$, the sideband suppression is higher than $20 \mathrm{~dB}$, and the output $P_{1 \mathrm{~dB}}$ is also higher than $-5 \mathrm{dBm}$. 


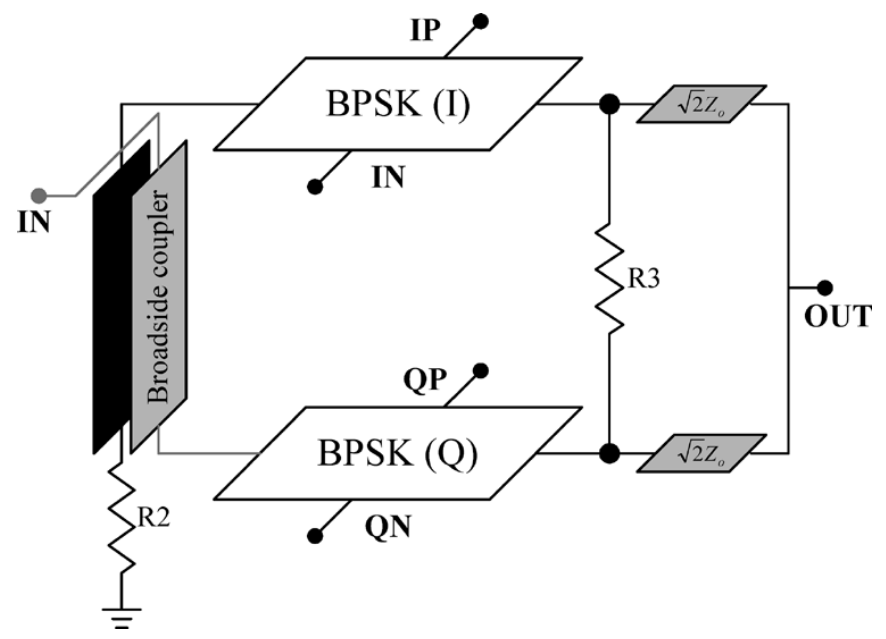

Fig. 9. Schematic of the modified reflection-type IQ modulator.

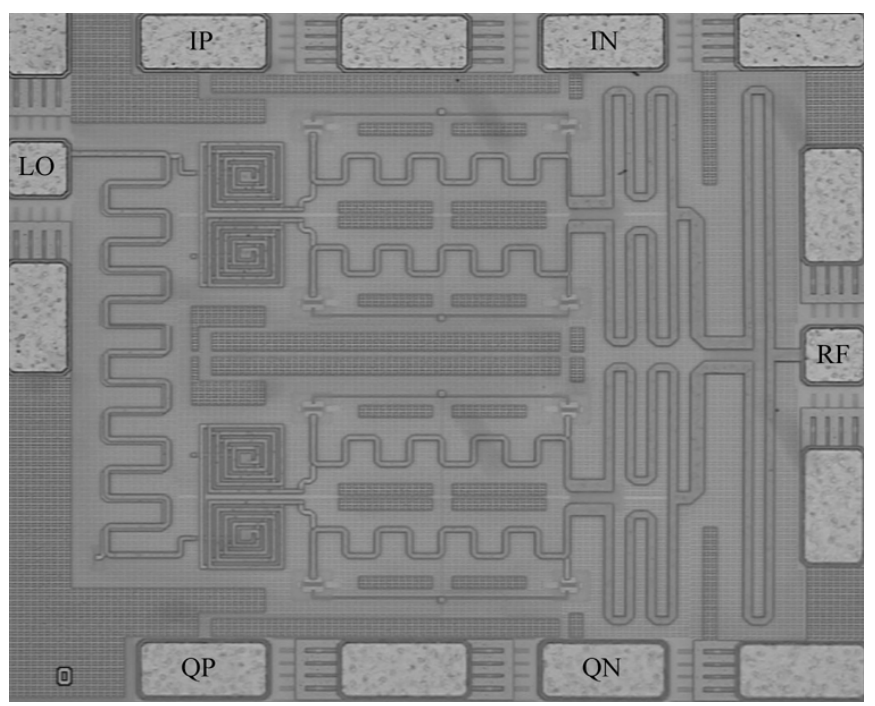

Fig. 10. Photograph of the modified reflection-type IQ modulator with a chip size of $0.65 \times 0.58 \mathrm{~mm}^{2}$.

\section{EXPERIMENTAL RESULTS}

\section{A. BPSK Modulator}

The measurements of the modulator chips are performed via on-wafer probing. The $S$-parameters of the chips were measured by using an Agilent $8510 \mathrm{C}$ network analyzer. The measured insertion losses of three states (states 0,1 , and OFF) between 1 to $80 \mathrm{GHz}$ for the modified reflection-type BPSK modulator are plotted in Fig. 11(a), and the amplitude and phase imbalances between states 0 and 1 are plotted in Fig. 11(b). The measured results agree well with the simulated results. From 15 to $75 \mathrm{GHz}$, the measured insertion losses are less than $13 \mathrm{~dB}$ and the isolation in the OFF state is better than $40 \mathrm{~dB}$, the amplitude imbalance is within $0.5 \mathrm{~dB}$, and the phase imbalance is with $3^{\circ}$. The worst case input and output return losses are better than $8 \mathrm{~dB}$.

For the conversion loss measurement of the BPSK modulator, an Agilent 83 650L signal generator with a broad-band amplifier is used for the LO, an Agilent E4438C microwave signal generator with a $180^{\circ}$ hybrid is used for baseband inputs, and the RF

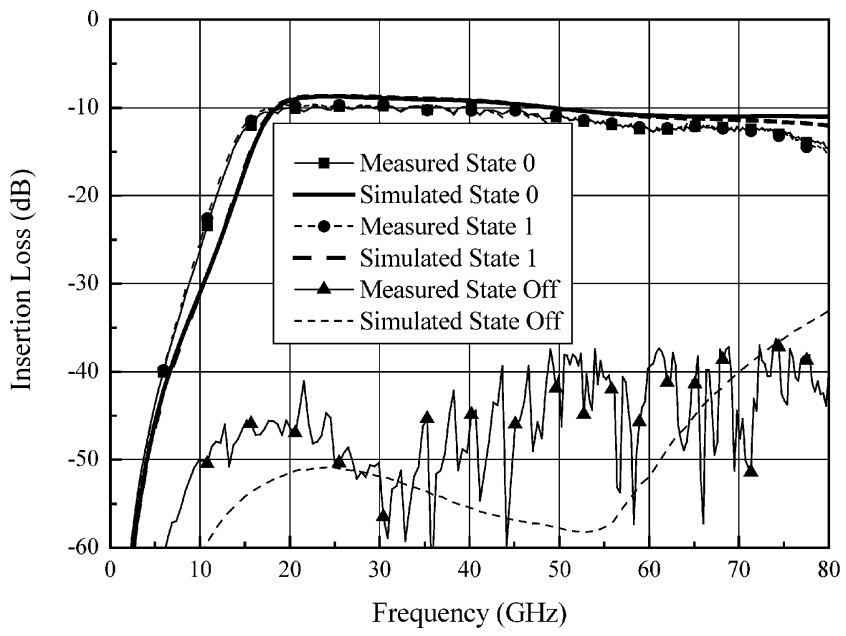

(a)

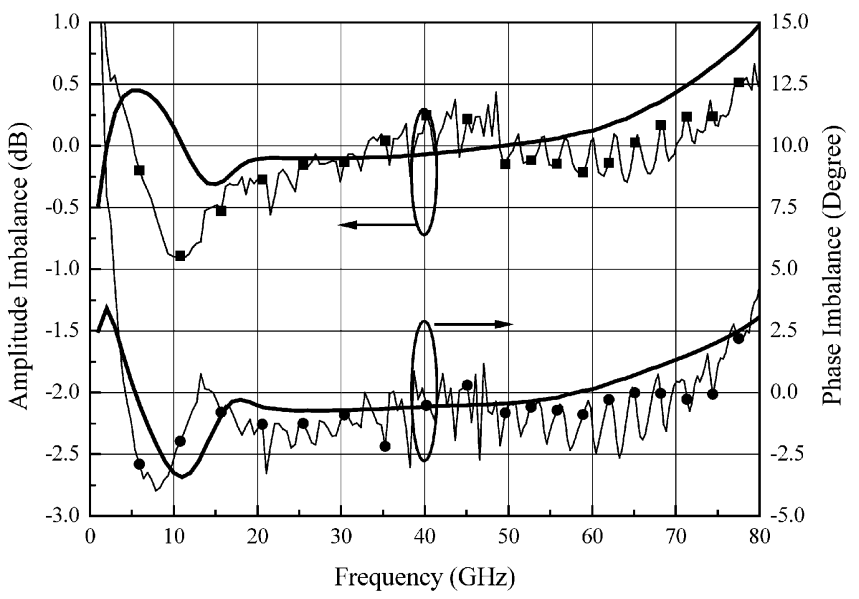

(b)

Fig. 11. Measured and simulated results of the modified reflection-type BPSK modulator. (a) Insertion losses of states 0, 1, and OFF. (b) Amplitude and phase imbalances between states 0 and 1 (simulation: solid line; measurement: symbol with line).

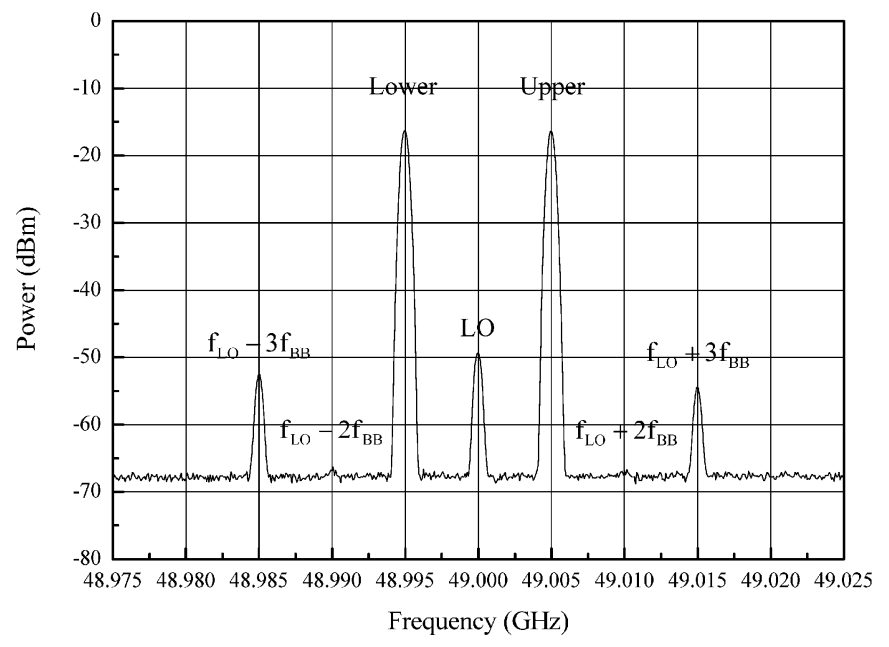

Fig. 12. Measured output spectrum of the BPSK modulator with the LO frequency of $49 \mathrm{GHz}$ and the baseband frequency of $5 \mathrm{MHz}$.

output signal is measured with an Agilent E4448A spectrum analyzer. The measured output spectrum of the BPSK modulator is plotted in Fig. 12, the LO source is $49 \mathrm{GHz}$ with a power 


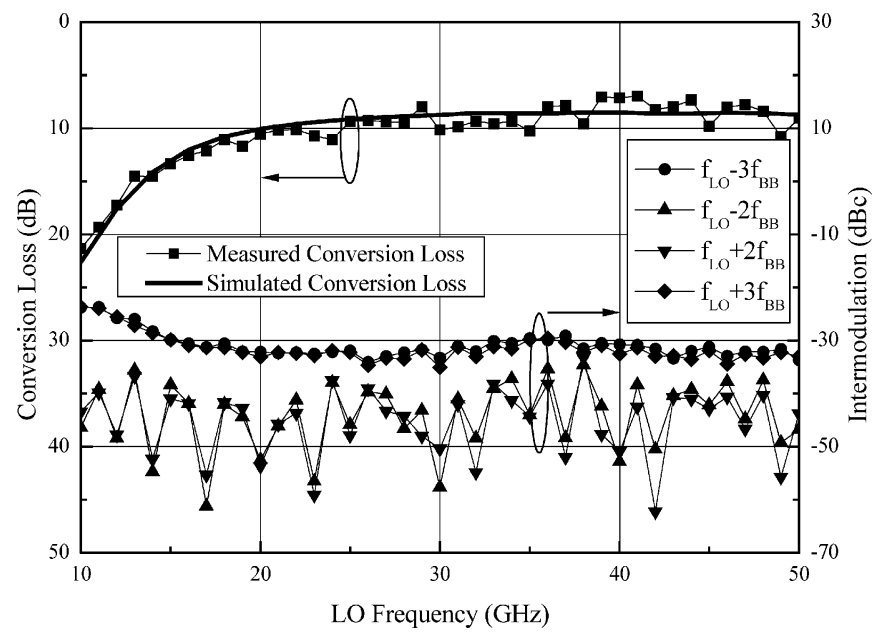

Fig. 13. Measured and simulated conversion loss and intermodulation versus the LO frequency from 10 to $50 \mathrm{GHz}$ for the BPSK modulator.

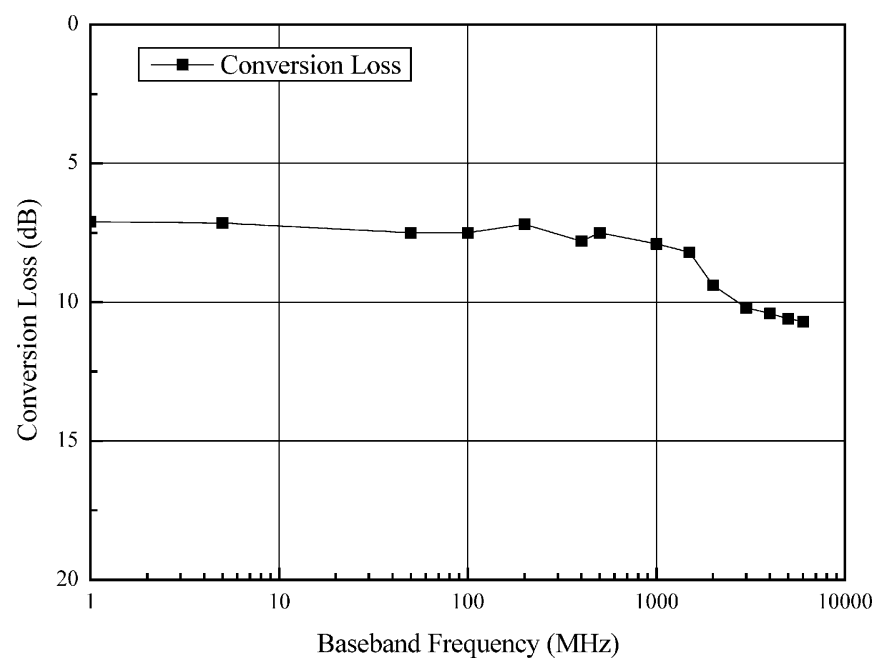

Fig. 14. Measured conversion loss versus the baseband frequency from $1 \mathrm{MHz}$ to $6 \mathrm{GHz}$ with an $\mathrm{LO}$ frequency of $40 \mathrm{GHz}$ for the BPSK modulator.

of $5 \mathrm{dBm}$, and the baseband signal is $5 \mathrm{MHz}$ with a power of $-8 \mathrm{dBm}$. The measured output $P_{1 \mathrm{~dB}}$ is higher than $-5 \mathrm{dBm}$ and is obtained from sweeping the baseband input power. The measured and simulated conversion loss and intermodulation ( $f_{\mathrm{LO}} \pm 2 f_{\mathrm{BB}}$ and $f_{\mathrm{LO}} \pm 3 f_{\mathrm{BB}}$ spurs) versus the LO frequency from 10 to $50 \mathrm{GHz}$ for the BPSK modulator is plotted in Fig. 13. It shows good agreement between measurement and simulation. Between 15 and $50 \mathrm{GHz}$, the measured conversion loss is better than $15 \mathrm{~dB}$, the second-order intermodulation $\left(f_{\mathrm{LO}} \pm 2 f_{\mathrm{BB}}\right)$ components are less than $-35 \mathrm{dBc}$ referred to the upper or lower sideband output power, and the third-order intermodulation $\left(f_{\mathrm{LO}} \pm 3 f_{\mathrm{BB}}\right)$ components are less than $-30 \mathrm{dBc}$. The measured conversion loss versus the baseband frequency from $1 \mathrm{MHz}$ to $6 \mathrm{GHz}$ with an $\mathrm{LO}$ frequency of $40 \mathrm{GHz}$ for the BPSK modulator is plotted in Fig. 14, which features a modulation bandwidth of greater than $1 \mathrm{GHz}$. Therefore, this modulator is suitable for wide-band digital modulation applications.

The modulation quality of the BPSK modulation is also measured and shown in [16], which demonstrates an error vector magnitude (EVM) of better than $3.5 \%$ and $7 \%$ for data rates of 1 and $10 \mathrm{Mb} / \mathrm{s}$, respectively, an adjacent channel power ratio

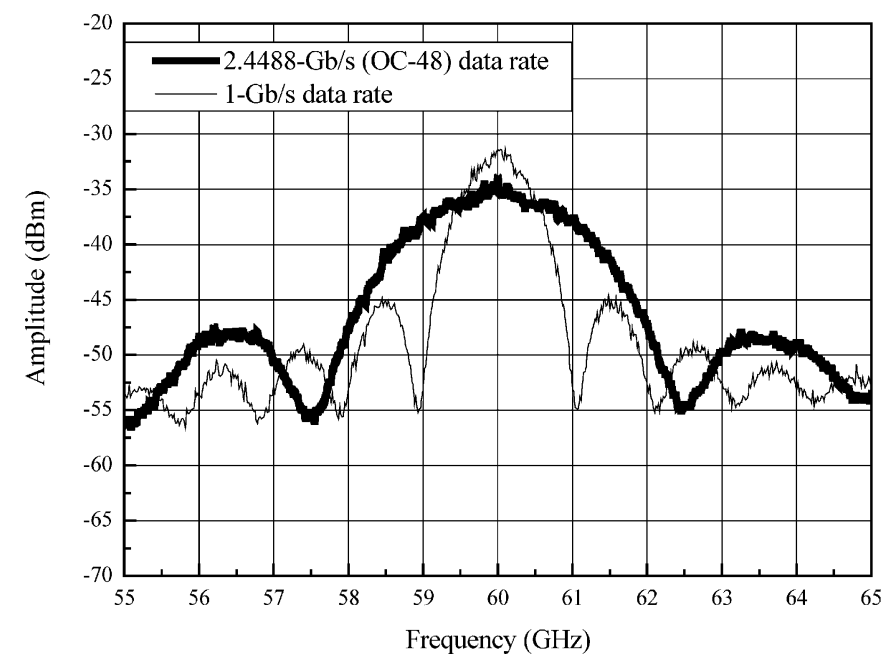

Fig. 15. Measured output spectrum of the BPSK modulator at $60 \mathrm{GHz}$ with 1and $2.4488-\mathrm{Gb} / \mathrm{s}(\mathrm{OC}-48)$ data rates in PRBS.

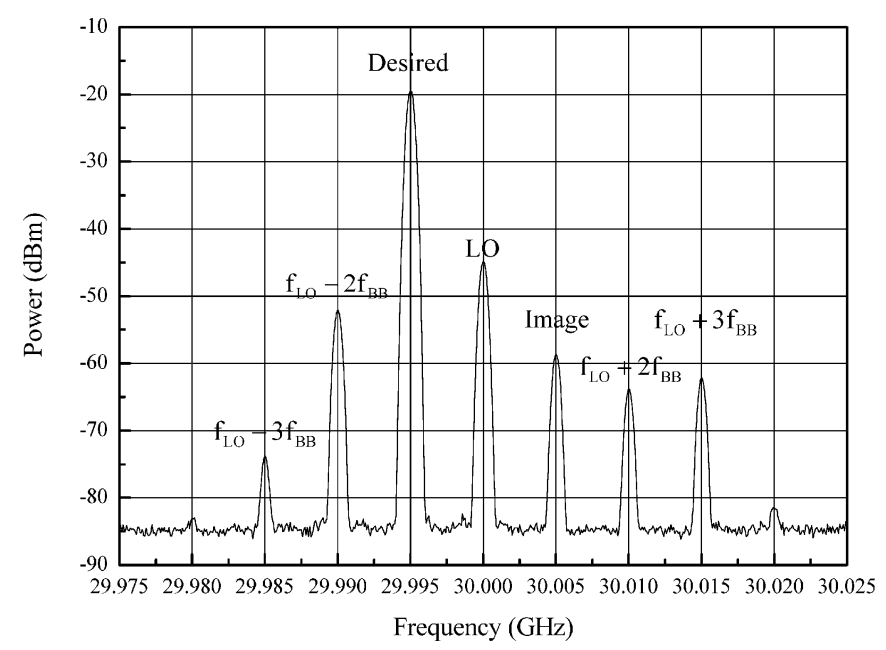

Fig. 16. Measured output spectrum of the IQ modulator with an LO frequency of $30 \mathrm{GHz}$ and a baseband frequency of $5 \mathrm{MHz}$.

(ACPR) of less than $-40 \mathrm{dBc}$, and an output channel power of higher than $-15 \mathrm{dBm}$. The BPSK modulator has also been evaluated by a bi-phase multilevel modulation, and the result shows good modulation quality, linearity, and ACPR. For wideband applications, the BPSK modulator is evaluated with 1- and $2.4488-\mathrm{Gb} / \mathrm{s}$ (OC-48 standard) data rates in a pseudorandom bit stream (PRBS). The differential baseband signals are generated from an Agilent pattern generator (N4901B series BERT), and the voltage swing of the baseband signal is from 0.2 to $0.8 \mathrm{~V}$. The LO is generated from an Agilent E8247C with an Agilent 83557A MMW source module, and the diver LO power is about $0 \mathrm{dBm}$. The measured output spectrum of the BPSK at $60 \mathrm{GHz}$ is plotted in Fig. 15, which features good LO suppression and a channel power of higher than $-20 \mathrm{dBm}$ for both data rates. The spectrum is spread out due to the unfiltered baseband signals, resulting in a sinc-like spectrum.

\section{B. IQ Modulator}

Fig. 16 shows the measured output spectrum of the IQ modulator with an $\mathrm{LO}$ frequency of $30 \mathrm{GHz}$ and a baseband frequency 


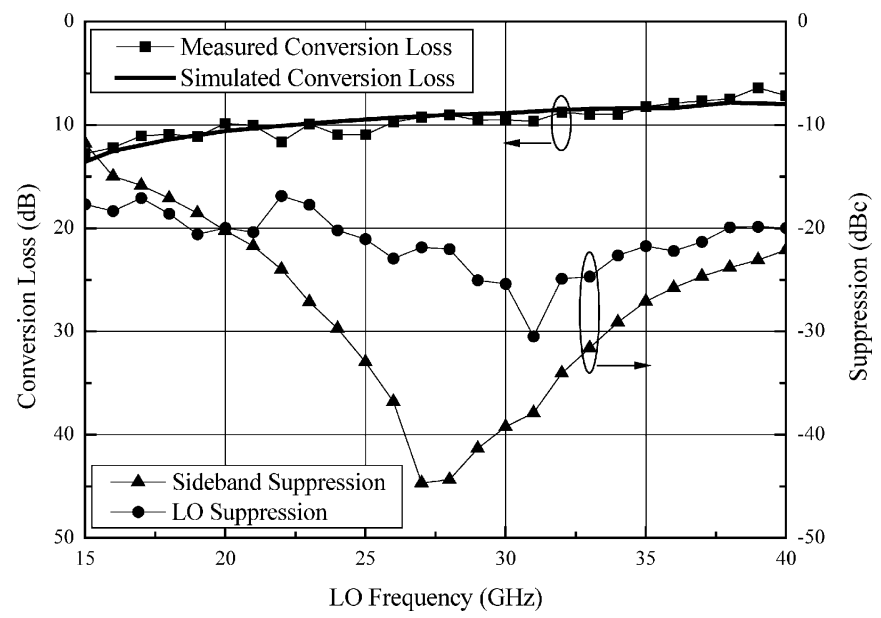

Fig. 17. Measured and simulated conversion loss, sideband, and LO suppressions versus the LO frequency from 15 to $40 \mathrm{GHz}$ for the IQ modulator.

of $5 \mathrm{MHz}$, where the desired output is the lower sideband (LSB) component and the image output is the upper sideband (USB) component. The measured output power of the modulator signal is $-19 \mathrm{dBm}$ when the baseband input of $-10 \mathrm{dBm}$ and the LO drive power of $10 \mathrm{dBm}$ are applied to the IQ modulator. The measured image suppression is $-39 \mathrm{dBc}$ and the measured LO suppression is $-25 \mathrm{dBc}$. The second-order intermodulation $\left(f_{\mathrm{LO}} \pm 2 f_{\mathrm{BB}}\right)$ components are less than $-33 \mathrm{dBc}$ referring to the desired output power (LSB), and the third-order intermodulation $\left(f_{\mathrm{LO}} \pm 3 f_{\mathrm{BB}}\right)$ components are less than $-44 \mathrm{dBc}$.

The measured output $P_{1 \mathrm{~dB}}$ is higher than $-10 \mathrm{dBm}$ with a sideband suppression of better than $-38 \mathrm{dBc}$ and an LO suppression of better than $-25 \mathrm{dBc}$. The spurs $\left(f_{\mathrm{LO}} \pm 2 f_{\mathrm{BB}}\right.$ and $\left.f_{\mathrm{LO}} \pm 3 f_{\mathrm{BB}}\right)$ suppressions are less than $-30 \mathrm{dBc}$ when the modulator is operated at output $P_{1} \mathrm{~dB}$. The measured and simulated conversion loss, sideband, and LO suppressions versus the LO frequency from 15 to $40 \mathrm{GHz}$ is plotted in Fig. 17 for the IQ modulator. The measured results also agree with the simulated results. Between 20 and $40 \mathrm{GHz}$, the measured sideband suppression is better than $-20 \mathrm{dBc}$ and the measured LO suppression is better than $-15 \mathrm{dBc}$. Since the low-imbalance (amplitude imbalance $<0.5 \mathrm{~dB}$ and phase imbalance $<1^{\circ}$ ) band of coupler 2 is narrow, the high sideband suppression $(<-40 \mathrm{dBc})$ occurs only from 27 to $30 \mathrm{GHz}$. The measured intermodulation $\left(f_{\mathrm{LO}} \pm 2 f_{\mathrm{BB}}\right.$ and $\left.f_{\mathrm{LO}} \pm 3 f_{\mathrm{BB}}\right)$ versus the LO frequency for the IQ modulator is plotted in Fig. 18, which demonstrates a second-order intermodulation of $-31 \mathrm{dBc}$ and a third-order intermodulation of $-38 \mathrm{dBc}$ from 20 to $40 \mathrm{GHz}$.

For the measurement of the modulation quality, an Agilent E8247C signal generator is used for the LO source because a good phase-noise source is required for the high-level modulation. An Agilent E4448A spectrum analyzer and an Agilent $86410 \mathrm{~A}$ vector signal analyzer are used for the vector signal characterization. The baseband signals are generated using Agilent ADS software and downloaded into an Agilent E4438C arbitrary waveform generator. The data frame is also constructed with a PRBS, and some additional digital filters are also utilized to minimize the spectrum spread. The LO drive power is about $10 \mathrm{dBm}$ and the baseband overall input power, including IP, IN, $\mathrm{QP}$, and QN, is about $-10 \mathrm{dBm}$. At an LO frequency of $30 \mathrm{GHz}$,

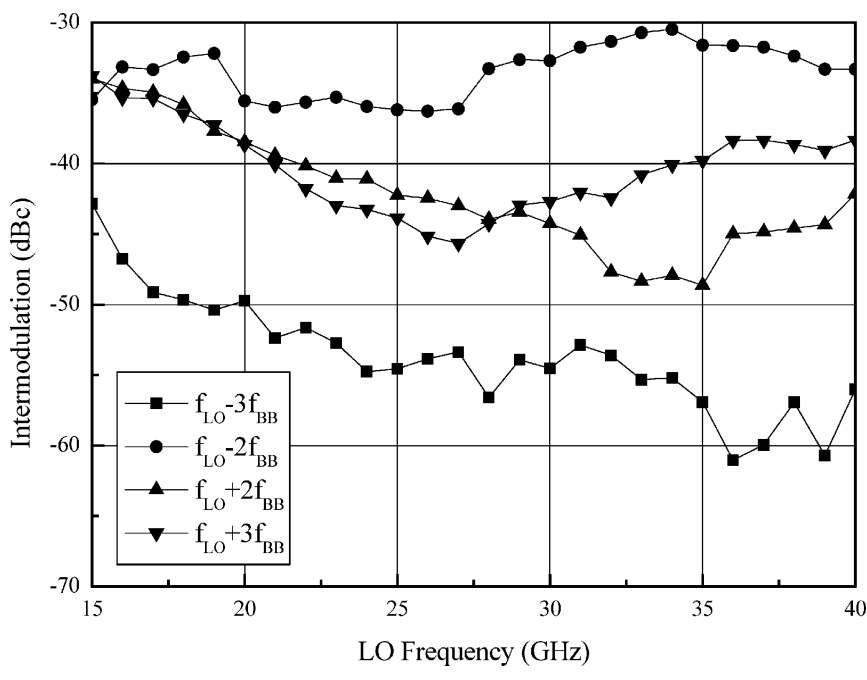

Fig. 18. Measured intermodulation versus the LO frequency from 15 to $40 \mathrm{GHz}$ for the IQ modulator.

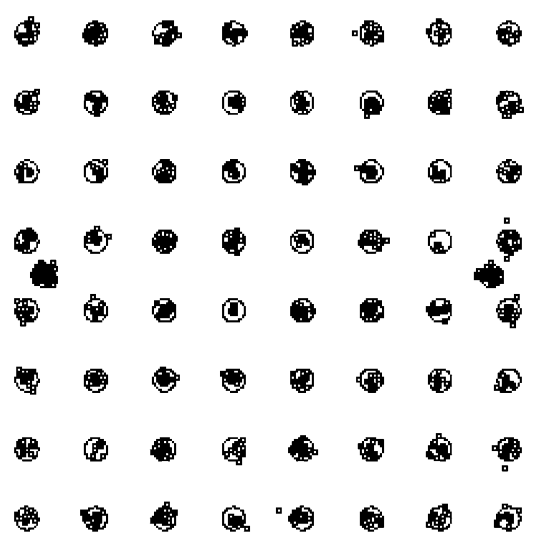

Fig. 19. Measured constellation diagram of the IQ modulator at $30 \mathrm{GHz}$ with a 54-Mb/s OFDM modulation (reference constellations: $\bigcirc$, recovery symbols: $\bullet$, EVM $<3 \%$, data: 64-QAM, pilot: BPSK).

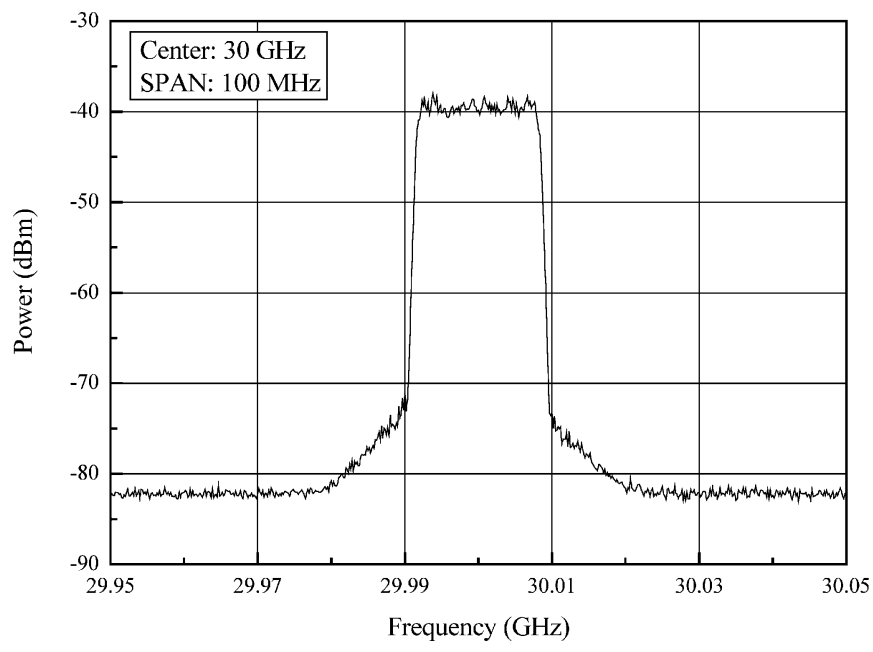

Fig. 20. Measured output spectrum of the IQ modulator at $30 \mathrm{GHz}$ with a $54-\mathrm{Mb} / \mathrm{s}$ OFDM modulation; the channel power is about $-18 \mathrm{dBm}$ with a channel bandwidth of $20 \mathrm{MHz}$.

the IQ modulator is evaluated by a 64-QAM modulation with a data rate of $6 \mathrm{Mb} / \mathrm{s}$. The measured modulation quality (EVM) of 
TABLE II

COMPARISON OF PREVIOUSLY REPORTEd CONVENTIONAL REFLECTION-TyPe BPSK/IQ MODULATORS AND THIS WORK

\begin{tabular}{|c|c|c|c|c|}
\hline & \multicolumn{2}{|c|}{ BPSK Modulator } & \multicolumn{2}{|c|}{ IQ Modulator } \\
\hline & Ref. [11]* & This work & Ref. [11]* & This work \\
\hline Frequency $(\mathrm{GHz})$ & $45-110$ & $15-75$ & $50-110$ & $20-40$ \\
\hline Modulation Bandwidth & $>10 \mathrm{MHz}$ & $>1 \mathrm{GHz}$ & $>10 \mathrm{MHz}$ & $>1 \mathrm{GHz}$ \\
\hline Conversion Loss (dB) & $<20$ & $<13 \mathrm{~dB} @ 15-50 \mathrm{GHz}$ & $<20$ & $<13 \mathrm{~dB} @ 15-40 \mathrm{GHz}$ \\
\hline Output $\mathrm{P}_{\mathrm{IdB}}(\mathrm{dBm})$ & $>-10$ & $>-5$ & $>-10$ & $>-5$ \\
\hline LO-to-RF Isolation (dB) & $>20$ & $>40$ & $>20$ & $>40$ \\
\hline $\mathrm{DC}$ offset $(\mathrm{dB})$ & $<-10$ & $<-30$ & $<-15$ & $<-30$ \\
\hline Sideband Suppression $(\mathrm{dBc})$ & --- & --- & $<-20 \mathrm{dBc} @ 55-95 \mathrm{GHz}$ & $\begin{array}{c}<-20 \mathrm{dBc} \\
<-40 \mathrm{dBc} @ 27-30 \mathrm{GHz}\end{array}$ \\
\hline Second-order Intermodulation $(\mathrm{dBc})$ & -25 & $<-35 \mathrm{dBc}$ & -25 & $<-30 \mathrm{dBc}$ \\
\hline Third-order Intermodulation $(\mathrm{dBc})$ & -20 & $<-30 \mathrm{dBc}$ & -20 & $<-30 \mathrm{dBc}$ \\
\hline Chip Size $\left(\mathrm{mm}^{2}\right)$ & $1 \times 1$ & $0.5 \times 0.35$ & $2 \times 2$ & $0.65 \times 0.58$ \\
\hline Device & GaAs HBT & CMOS & GaAs HBT & CMOS \\
\hline
\end{tabular}

* With calibration bias and DC offset compensation

64-QAM is less than $1.8 \%$ with a phase error of within $1^{\circ}$ and an amplitude error of within $1 \%$. The measured ACPR is better than $-50 \mathrm{dBc}$ with a channel power of higher than $-18 \mathrm{dBm}$. For wide-band applications, the IQ modulator is also evaluated with a 54-Mb/s OFDM modulation signal. The measured constellation diagram of the IQ modulator at $30 \mathrm{GHz}$ is plotted in Fig. 19, which shows a 64-QAM constellation for data signals and a BPSK constellation for pilot signals. The measured EVM of the OFDM modulation is within 3\%. The measured output spectrum of the IQ modulator with a 54-Mb/s OFDM modulation is plotted in Fig. 20, which demonstrated a channel power of higher than $-18 \mathrm{dBm}$ with a channel bandwidth of $20 \mathrm{MHz}$ and an ACPR of better than $-40 \mathrm{dBc}$.

The comparison of previously reported conventional reflection-type BPSK/IQ modulators and this work are summarized in Table II. The modified reflection-type modulators in this paper demonstrate broad RF bandwidth, gigabit modulation bandwidth, good LO-to-RF isolation, high linearity, and ultracompact chip sizes. Compared to the prior work based on GaAs HBT technology [11], this work exhibits better modulation bandwidth, conversion loss, LO-to-RF isolation, and secondand third-order intermodulations and smaller chip size due to the innovative topology and CMOS characteristics.

\section{CONCLUSION}

An innovative topology for the reflection-type modulator is proposed and analyzed in this paper, and the modified reflection-type BPSK and IQ modulators are successfully implemented using standard bulk $0.13-\mu \mathrm{m}$ CMOS process with ultra compact chip size. Compared with the conventional reflection-type modulators, these modulators demonstrated low amplitude/phase imbalances and high LO-to-RF isolation (or low dc offset) with broad bandwidth due to the proposed topology. The BPSK modulator demonstrates an RF frequency of $15-75 \mathrm{GHz}$ with good amplitude/phase match. For the IQ modulator, the RF frequency is from 20 to $40 \mathrm{GHz}$, and it features good sideband, LO, and spur suppressions. The modulators also achieve low conversion loss, gigabit modulation bandwidth, and high linearity. Therefore, they can be further applied to ultrawide-band transmitter applications. Due to low cost and mass production of the standard bulk CMOS process, it is very attractive for the system integration including digital signal process and front-end RF circuits in a single chip. From this demonstration, the CMOS technology has potential applications in the MMW regime.

\section{ACKNOWLEDGMENT}

The authors gratefully acknowledge the Taiwan Semiconductor Manufacturing Company, Hsinchu City, Taiwan, R.O.C., for the chips fabrication. The authors would also to like to thank Prof. G. D. Vendelin and M.-F. Lei, National Taiwan University, Taipei, Taiwan, R.O.C., for the discussions, and P. Lin, Taiwan Agilent, Taipei, Taiwan, R.O.C., for help with the high-speed data rate measurement.

\section{REFERENCES}

[1] C.-Y. Wu and H.-S. Kao, "A 2-V low-power CMOS direct-conversion quadrature modulator with integrated quadrature voltage-controlled oscillator and RF amplifier for GHz RF transmitter applications," IEEE Trans. Circuit Syst. I, Reg. Papers, vol. 49, pp. 123-134, Feb. 2002.

[2] N. Sornin, M. Massel, L. Perraud, and C. Pinatel, "A robust Cartesian feedback loop for a $802.11 \mathrm{a} / \mathrm{b} / \mathrm{g}$ CMOS transmitter," in IEEE RFIC Symp. Dig., Fort Worth, TX, Jun. 2004, pp. 145-148.

[3] K.-Y. Lee, S.-W. Lee, Y. Koo, H.-K. Huh, H.-Y. Nam, J.-W. Lee, J. Park, K. Lww, D.-K. Jeong, and W. Kim, "Full-CMOS 2-GHz WCDMA direct conversion transmitter and receiver," IEEE J. Solid-State Circuits, vol. 38, no. 1, pp. 43-53, Jan. 2003.

[4] P. Zhang, T. Nguyen, C. Lam, D. Gambetta, C. Soorapanth, B. Cheng, S Hart, I. Sever, T. Bourdl, A. Tham, and B. Razavi, "A direct conversion CMOS transceiver for IEEE 802.11a WLANs," in IEEE ISSCC Dig., Feb. 2003, pp. 354-355.

[5] R. Ahola, A. Aktas, J. Wilson, K. R. Rao, F. Jonsson, H. Isto, A. Brolin, T. Hakala, A. Friman, M. Tuula, J. Hanze, S. Martin, D. Wallner, Y. Guo, T. Lagerstam, L. Noguer, T. Knuuttila, P. Olofsson, and M. Ismail, "A single-chip CMOS transceiver for $802.11 \mathrm{a} / \mathrm{b} / \mathrm{g}$ wireless LANs," IEEE J. Solid-State Circuits, vol. 39, no. 12, pp. 2250-2258, Dec. 2004.

[6] K. Nakajima, T. Sugano, and N. Suematsu, "A 5 GHz-band SiGe-MMIC direct quadrature modulator using a doubly stacked polyphase filter," in IEEE RFIC Symp. Dig., Fort Worth, TX, Jun. 2004, pp. 409-412.

[7] S. A. Maas, The RF and Microwave Circuit Design Cookbook. Boston, MA: Artech House, 1998.

[8] D. C. W. Lo, H. Wang, B. R. Allen, G. S. Dow, K. W. Chang, M. Biedenbender, R. Lai, S. Chen, and D. Yang, "Novel monolithic multifunctional balanced switching low-noise amplifiers," IEEE Trans. Microw. Theory Tech., vol. 42, no. 12, pp. 2629-2634, Dec. 1994. 
[9] T. Lodhi, D. L. Edgar, H. McLelland, S. Ferguson, K. Elgaid, C. R. Stanley, and I. G. Thayne, "A $77 \mathrm{GHz}$ coplanar waveguide MMIC BPSK vector modulator realized using InP technology," in IEEE Gallium Arsenide Integrated Circuit Symp. Dig., 2000, pp. 183-186.

[10] D. S. McPherson and S. Lucyszyn, "Vector modulator for $W$-band software radar techniques," IEEE Trans. Microw. Theory Tech., vol. 49, no. 8, pp. 1451-1461, Aug. 2001.

[11] H.-Y. Chang, T.-W. Huang, H. Wang, Y.-C. Wang, P.-C. Chao, and C.-H. Chen, "Broad-band HBT BPSK and IQ modulator MMICs and millimeter-wave vector signal characterization," IEEE Trans. Microw. Theory Tech., vol. 52, no. 3, pp. 908-919, Mar. 2004.

[12] Y. Sun and A. P. Freundorfer, "A new overlay coupler for direct digital modulator in GaAs HBT," IEEE Trans. Microw. Theory Tech., vol. 52, no. 8, pp. 1830-1835, Aug. 2004.

[13] H.-Y. Chang, P.-S. Wu, T.-W. Huang, H. Wang, Y.-C. Tsai, and C.-H. Chen, "An ultra compact and broadband $15-75 \mathrm{GHz}$ BPSK modulator using 0.13- $\mu \mathrm{m}$ CMOS process," in IEEE MTT-S Int. Microwave Symp. Dig., Long Beach, CA, Jun. 2005, pp. 41-44.

[14] L. M. Franca-Neto, R. E. Bishop, and B. A. Bloechel, "64 GHz and 100 GHz VCO's in $90 \mathrm{~nm}$ CMOS using optimum pumping method," in IEEE ISSCC Dig., Feb. 2003, pp. 444-445.

[15] Sonnet User's Manual, Release 9.0, Sonnet Software Inc., North Syracuse, NY, May 2003.

[16] Y. Fujiki, H. Mandai, and T. Morikawa, "Chip type spiral broadside coupled directional couplers and baluns using low temperature co-fired ceramic," in Electric Components and Technology Conf., Jun. 1999, pp. $105-110$

[17] P.-S. Wu, C.-H. Wang, T.-W. Huang, and H. Wang, "Compact and broadband millimeter-wave monolithic transformer balanced mixers," IEEE Trans. Microw. Theory Tech., vol. 53, no. 10, pp. 3106-3114, Oct. 2005.

[18] H.-C. Lu and T.-H. Chu, "Port reduction methods for scattering matrix measurement of an n-port network," IEEE Trans. Microw. Theory Tech., vol. 48, no. 6, pp. 959-968, Jun. 2000.

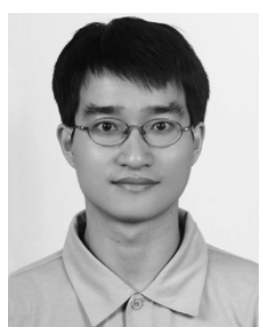

Hong-Yeh Chang ( $\left.\mathrm{S}^{\prime} 02-\mathrm{M}^{\prime} 05\right)$ was born in Kinmen, Taiwan, R.O.C., in 1973. He received the B.S. and M.S. degrees in electric engineering from National Central University, Chung-Li, Taiwan, R.O.C., in 1996 and 1998 respectively, and the Ph.D. degree in communication engineering from National Taiwan University, Taipei, Taiwan, R.O.C., in 2004.

In 1998, he joined Chunghwa Telecom Laboratories, Taoyuan, Taiwan, R.O.C., where he was involved with the research and development of code-division multiple-access (CDMA) cellular phone systems. From 1999 to 2000, he was with Syncomm Inc., Taoyuan, where he was invovled with the personal access communications system (PACS) radio port and handset design. He is currently a Post-Doctoral Research Fellow with the Graduate Institute of Communication Engineering, National Taiwan University. His research interests include monolithic microwave and millimeter-wave integrated circuit designs, transceiver and power amplifier linearization, and RF signal measurement.

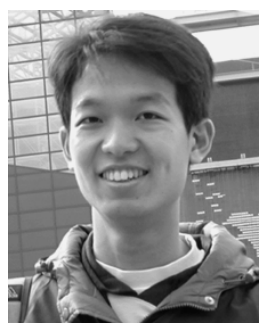

Pei-Si Wu (S'02) was born in Changhua, Taiwan, R.O.C., in 1980. He received the B.S. degree in electric engineering from National Taiwan University, Taipei, Taiwan, R.O.C., in 2002, where he is currently working toward the Ph.D. degree.

His research interests include microwave and millimeter-wave circuit designs.

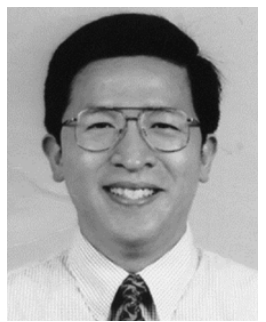

Tian-Wei Huang (S'91-M'98-SM'02) received the B.S. degree from National Cheng Kung University, Tainan, Taiwan, R.O.C., in 1987, and the M.S. and $\mathrm{Ph} . \mathrm{D}$. degrees from the University of California at Los Angeles (UCLA), in 1990 and 1993, respectively, all in electrical engineering.

In 1993, he joined the TRW RF Product Center, Redondo Beach, CA. His research has focused on the design and testing of monolithic microwave integrated circuits (MMICs) and RF integrated circuits (RFICs). During 1998-1999, he was with Lucent Technologies, working on the local multipoint distribution system fixed wireless systems. From 1999 to 2002, he was involved with the RF/wireless system testing at Cisco Systems. In August 2002, he joined the faculty of the Department of Electrical Engineering, National Taiwan University, Taipei, Taiwan, R.O.C. His current research interests are MMIC/RFIC design, packaging, and RF system-on-chip integration.

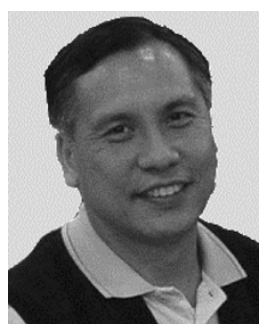

Huei Wang (''83-M'87-SM'95-F'06) was born in Tainan, Taiwan, R.O.C., on March 9, 1958. He received the B.S. degree in electrical engineering from National Taiwan University, Taipei, Taiwan, R.O.C., in 1980, and the M.S. and Ph.D. degrees in electrical engineering from Michigan State University, East Lansing, MI, in 1984 and 1987, respectively.

During his graduate study, he was engaged in research on theoretical and numerical analysis of electromagnetic radiation and scattering problems. $\mathrm{He}$ was also involved in the development of microwave remote detecting/sensing systems. In 1987, he joined the Electronic Systems and Technology Division, TRW Inc. (now Northrop Grumman), Redondo Beach, CA. He has been an MTS and Staff Engineer responsible for MMIC modeling of computer-aided design (CAD) tools, and MMIC testing evaluation and design, and became the Senior Section Manager of the Millimeter-Wave Sensor Product Section, RF Product Center. In 1993, he visited the Institute of Electronics, National Chiao-Tung University, Hsinchu City, Taiwan, R.O.C., where he taught MMIC-related topics. In 1994, he returned to TRW Inc. In February 1998, he joined the faculty of the Department of Electrical Engineering, National Taiwan University, Taipei, Taiwan, R.O.C., where he is currently a Professor.

Dr. Wang is a member of Phi Kappa Phi and Tau Beta Pi. He was the recipient of the Distinguished Research Award presented by the National Science Council, R.O.C. (2003-2006). He was also elected as the first Richard M. Hong Endowed Chair Professor of National Taiwan University in 2005.

Chung-Long Chang received the B.S. degree in electrical engineering from the Feng-Chia University, Taichong, Taiwan, R.O.C., in 1995.

He joined the Taiwan Semiconductor Manufacturing Company, Hsinchu City, Taiwan, R.O.C., in 1995. From 1995 to early 2000, he was involved with the Advanced Module Division's Dielectric \& CMP Department. In early 2000, he joined the Logic Technology Division, where he was invovled with mixed-mode RF groups, and he is now a Section Manager of the Communication Technology Department. He is the champion for MIM/MOM capacitors development including the coordination of RD and FAB module teams, process and product qualification, and technology transfer.

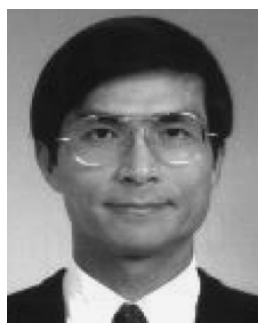

John G. J. Chern received the B.S. degree from the National Taiwan University, Taipei, Taiwan, R.O.C., in 1975, and the M.S. and Ph.D. degrees from the University of California, Berkeley, in 1979 and 1984, respectively, all in electrical engineering.

$\mathrm{He}$ has been involved with the IC industry throughout his career. His experiences include CMOS and BiCMOS process development, process/device simulation tools development, and EPROM/DRAM/SRAM device design and process development. He joined Taiwan Semiconductor Manufacturing Company, Hsinchu City, Taiwan, R.O.C., in 1993 and is now a Director in Research and Development to lead the development of communication technology including mixed-signal RF CMOS and SiGe BiCMOS. 